\title{
Fluorine F 18 Clofarabine
}

National Cancer Institute

\section{Source}

National Cancer Institute. Fluorine F18 Clofarabine. NCI Thesaurus. Code C126106.

A radioconjug ate composed of the nucleoside analog and deoxycytidine kinase (DCK)dependent pro-drug clofarabine ( $\mathrm{Cl}-\mathrm{F}$-ara-A) linked to the radioisotope fluorine $\mathrm{F} 18$, with potential imaging activity using positron emission tomography/computed tomography (PET/CT). Upon administration of fluorine F 18 clofarabine, the clofarabine moiety is preferentially taken up by and accumulates in cells with dysregulated nucleoside metabolism, including tumor cells expressing high levels of DCK. The clofarabine moiety is phosphorylated by DCK into its active triphosphate form, Cl-F-ara-ATP. The $18 \mathrm{~F}$ moiety can be visualized by PET imaging. As many nucleoside analog prodrugs are chemotherapeutic agents that require DCK for their phosphorylation and activation, fluorine F 18 clofarabine can potentially be used as a marker to measure DCK activity and to predict the chemotherapeutic efficacy of DCK-dependent prodrugs. DCK, a ratelimiting enzyme in the deoxyribonucleoside salvage pathway for DNA synthesis, is overexpressed in certain solid tumors, lymphoid and myeloid malignancies and certain immune cells, such as proliferating T-lymphocytes. Compared to other nucleoside analogs, clofarabine is not susceptible to deamination by cytidine deaminase (CDA). 